\title{
Does emergency presentation of cancer represent poor performance in primary care? Insights from a novel analysis of linked primary and secondary care data
}

\author{
Peter Murchie ${ }^{\star}, 1$, Sarah M Smith ${ }^{1}$, Michael S Yule ${ }^{1}$, Rosalind Adam ${ }^{1}$, Melanie E Turner ${ }^{1}$, Amanda J Lee ${ }^{1}$ \\ and Shona Fielding ${ }^{1}$ \\ ${ }^{1}$ Institute of Applied Health Sciences, University of Aberdeen, Polwarth Building, Foresterhill, Aberdeen AB25 2ZD, UK
}

\begin{abstract}
Background: People diagnosed with cancer following emergency presentation have poorer short-term survival. To what extent this signifies a missed opportunity for earlier diagnosis in primary care remains unclear as little detailed data exist on the patient/ general practitioner interaction beforehand.
\end{abstract}

Methods: Analysis of primary care and regional data for 1802 cancer patients from Northeast Scotland. Adjusted odds ratios (OR) and $95 \%$ confidence intervals (Cls) for patient and GP practice predictors of emergency presentation. Qualitative context coding of primary care interaction before emergency presentation.

Results: Emergency presentations equalled 20\% $(n=365)$. Twenty-eight per cent had no relevant prior GP contact. Of those with prior GP contact 30\% were admitted while waiting to be seen in secondary care, and 19\% were missed opportunities for earlier diagnosis. Associated predictors: no prior GP contact $(\mathrm{OR}=3.89$; $\mathrm{Cl} 95 \%$ 2.14-7.09); having lung ( $\mathrm{OR}=23.24 ; 95 \% \mathrm{Cl} 7.92-68.21)$, colorectal $(\mathrm{OR}=18.49 ; \mathrm{Cl} 95 \%$ 6.60-51.82) and upper $\mathrm{Gl}$ cancer ( $\mathrm{OR}=18.97$; $\mathrm{Cl} 95 \%$ 6.08-59.23); ethnicity $(\mathrm{OR}=2.78 ; \mathrm{Cl} 95 \%$ 1.27-6.06).

Conclusions: Our novel approach has revealed that emergency cancer presentation is more complex than previously thought. Patient delay, prolonged referral pathways and missed opportunities by GPs all contribute, but emergency presentation can also represent effective care. Resources should be used proportionately to raise public and GP awareness and improve post-referral pathways.

In England, a fifth of all cancers (20\%) are diagnosed following emergency presentation (EP) to a general practitioner (GP) or hospital emergency department (National Cancer Intelligence Network, 2015). Studies on large clinical data sets consistently show that EP is predicted by older age, gender, socioeconomic deprivation and cancer type, more frequently brain and lung (McPhail et al, 2013; Abel et al, 2015; Mitchell et al, 2015a; National Cancer Intelligence Network, 2015) and that, following adjustment for age, stage and co-morbidity, emergency cancer patients have poorer short-term survival compared with patients diagnosed through other routes (Elliss-Brookes et al, 2012; McPhail et al, 2013; National Cancer Intelligence Network, 2015). Studies in Scotland have made similar findings for people with breast and colorectal cancer (McArdle and Hole, 2004; Brewster et al, 2011). Previous research has reported that most patients with an emergency cancer presentation have had contact with a GP in the months or weeks beforehand (Barrett and Hamilton, 2008; Lyratzopoulus et al, 2012; Mitchell et al, 2015b; Renzi et al, 2016; Wilcock et al, 2016). Similar findings have been widely described in the media, for example, in The Telegraph

*Correspondence: Dr P Murchie; E-mail: p.murchie@abdn.ac.uk

Received 21 October 2016; revised 20 February 2017; accepted 28 February 2017; published online 23 March 2017

(C) 2017 Cancer Research UK. All rights reserved 0007-0920/17 
newspaper, 'Tens of thousands of cancer patients visiting GP multiple times before referral' (Collins, 2013), and on the BBC website (2016) 'Alarm symptoms missed in bowel cancer emergency patients'. EP is also reported to be more likely for patients registered at GP practices with poor quality and outcomes framework (QoF) performance, more non-UK qualified GPs, and fewer $48 \mathrm{~h}$ appointments, although whether the subjects in this large data set had actually been seen by a GP prior to their EP was not determined (Bottle et al, 2012). These findings admit the possibility that errors and missed opportunities in primary care could be one of the root causes of patients presenting as cancer emergencies.

However, work to date on EP has been mainly based on large data sets employing routinely collected health-care data to provide a high-level overview of the patient, the disease and general practice characteristics associated with emergency cancer presentation. These data do improve the understanding of EP, but it gives relatively little insight into the nature of patient interactions with primary care in the lead up to EP, or the context and content of primary care consultations during which GP decisions are made. Recently, this has been partly addressed in an important paper by Renzi et al (2016) who studied EP of colorectal cancer using routine primary data from the Clinical Practice Research Datalink including information about the number of primary contacts and the associated symptom codes. This is a considerable step forward but does not enable an exploration of what has actually occurred in the interactions between patients and their GPs in the lead up to an emergency cancer presentation. It is this contextual detail that is crucial to a true understanding of the causes and implications of emergency cancer presentation. Appropriate interventions and policy initiatives to help improve EP survival can only follow from this knowledge (McPhail et al 2013; Rubin et al 2013).

We explored the role of primary care in emergency cancer presentation for people diagnosed with six common cancers in Northeast Scotland (breast, colorectal, lung, melanoma, prostate and upper gastro-intestinal (GI)). For each patient, we linked a comprehensive primary care data set to secondary care data held on NHS Grampian's secondary care Cancer Care Pathway database (CCPd), to form a uniquely detailed clinical data set. We believe this is the first study to explore EP of six common cancers using this method, and to provide important new insights into the issue of emergency cancer presentation.

\section{MATERIALS AND METHODS}

Patient sample size. The study formed part of an evaluation in Northeast Scotland of the impact of the Scottish Government's 'Detect Cancer Early' (DCE) campaign and the effectiveness of the way Scottish Referral Guidelines for Suspected Cancer (SRGSC) are used. The cancers chosen were the six commonest in the region and included those in the DCE campaign (breast, colorectal and lung). We calculated a conservative sample size based on the most common (breast) and the least common (melanoma) to inform the minimum number of cases to include in our data collection samples to give enough power to estimate meaningful differences between relative cancer diagnostic pathways.

GP practice recruitment. All general practices in NHS Grampian, Orkney and Shetland $(n=101)$ were invited to participate and 55 practices (54\%) agreed. Thirty-five practices were randomly selected, stratified by list size, location and deprivation (Scottish Government, 2012). Using the NHS Grampian CCPd, 2500 patients with one of the six common cancers, and registered at the selected 35 practices, were randomly selected for a detailed case-note review. Thirty-one practices were actually visited between October 2013 and January 2015, and 2102 case-notes were reviewed of patients diagnosed with cancer from 2007 to 2013. Four practices could not accommodate a visit from the study researcher within the allowed timescale.

\section{Data collection}

Detailed data from primary care electronic records. Using a predefined data extraction form, for all cases where available, the date of each sequential event leading to diagnosis was recorded, that is, symptom onset; presentation; referral/admission; hospital appointment; diagnosis and treatment. Date of death was recorded where appropriate. Details were also collected on sociodemographic and lifestyle characteristics; route to diagnosis; symptoms and outcomes; relevant investigations before referral; co-morbidities; current prescriptions; treatment type; stage at diagnosis and number of relevant consultations before the referral that led to diagnosis.

Regional data. The NHS Grampian CCPd was used to provide data on secondary care appointments, investigations and treatment received for all people diagnosed with cancer in Northeast Scotland whatever their route to referral.

Data management. Primary care records were collected using Microsoft Access, and the NHS Grampian CCPd data were available in Microsoft Excel. Both files were exported into SPSS version 23.0 and linked using the patients' Community Health Index (CHI) number (NHS National Services Scotland, 2016). CHI numbers were then removed leaving a unified anonymous file for analysis.

Data analysis. Patients whose cancer was diagnosed through breast or bowel screening were excluded from analysis.

For each patient, all primary care presentations in the 24 months prior to the referral or admission that led to diagnosis were read in their entirety. Each relevant consultation was then examined to ascertain whether GPs had appropriately followed referral advice given in the Scottish Referral Guidelines for Suspected Cancer (SRGSC; Scottish Executive Health Department, 2009). 'Relevant' consultations were those that included any clinical signs and symptoms in the SRGSC that recommended investigation (e.g., a chest X-ray) or an urgent suspected cancer (USC) referral. To identify those that were emergency admissions, cases were categorised by route to diagnosis and then divided into non-EP and EP.

EP was defined as:

(1) A presentation to primary care (including out of hours care) resulting in a same day emergency hospital admission and a subsequent cancer diagnosis. or

(2) A presentation directly to an accident and emergency (A\&E) department which resulted in a cancer diagnosis.

All other routes to diagnosis were classed as non-EPs.

Patient and practice predictors of EP. Univariate logistic regression analysis was performed for each characteristic with outcome (EP) to obtain unadjusted odds ratios. Any independent predictor from the univariate analysis found to be significant at the conservative $P \leqslant 0.10$ level (to avoid exclusion of borderline significant predictors) was included in the multivariate regression model.

Descriptive exploration of interaction with primary care before $E P$. A coding framework, to explore the interaction with primary care before EP, was devised by PM and agreed with SMS (the researcher who collected the data) (Box 1). Account was taken of 
any related referrals and/or linked investigations prior to $\mathrm{EP}$, as well as the effect of consulting behaviour on EP. Each EP was then coded by SMS to reflect this exploration. A 10\% quality assurance check was conducted by two clinical authors, PM and RA, who independently confirmed that SMS was applying the coding framework consistently.

Ethics. Anonymised data were collected for this study as part of the 'Detect Cancer Early' project, an NHS Grampian service improvement exercise. The study was reviewed by the Caldicott Guardian for NHS Grampian and approved by the North of Scotland Research Ethics Committee, who confirmed that the project was a service evaluation and that there was no requirement to contact individual patients prior to review of their medical records.

\section{RESULTS}

GP practice characteristics. Table 1 shows that the randomly selected group of participating GP practices contained an even spread of small, medium and large practices; a greater female to male GP ratio $(n=20,65 \%)$; and more urban practices and fewer deprived practices, which is reflective of the general practice makeup of Northeast Scotland. In comparison, non-participating practices were mainly rural with small list sizes and a more even spread of male and female GPs, and, as in participating practices, had fewer deprived patients.

Included cases. Of the 2102 case notes reviewed, 300 patients whose cancer was diagnosed as a result of breast or bowel screening were excluded, leaving 1802 records in the EP analysis.

Route to diagnosis. Taking all six cancers together, the commonest route to diagnosis was through GP USC or urgent referral $(n=1031,58 \%)$; non-urgent referrals and other routes accounted for $22 \%(n=406)$. EP occurred in $20 \%$ of cases $(n=365$; Table 2$)$. Colorectal, lung and upper GI cancers were the cancer types predominantly diagnosed through this route, compared with breast, prostate and melanoma and non-EPs (Table 2).

Sociodemographic characteristics of emergency and non-emergency patients. In contrast with non-emergency patients, patients presenting as emergencies tended to be older, with more current smokers and fewer never-smokers, slightly more deprived and with more patients of non-white ethnicity. These data are shown in Table 3, which also includes the demographics of patients within key EP subcategories.

\section{Box 1. Context coding of emergency presentation (EP)}

- EP to primary care with no relevant prior contact.

- EP to A\&E with no relevant prior primary care contact.

- EP occurred within an appropriate episode of care (e.g., patients who initially presented with non-alarm symptoms, patients who had relevant investigations before referral).

- EP occurred while awaiting a relevant secondary care appointment.

- EP occurred following a missed opportunity for earlier investigation (i.e., GPs had not adhered to Scottish Referral Guidelines for Suspected Cancer).

- EP occurred after non-attendance of follow-up appointment/long periods between relevant consultations.

- EP followed patient reluctance/refusal for secondary care investigation

- EP to A\&E followed previous relevant primary care contact.
Predictors of EP compared with all presentations. The detailed results of unadjusted analysis including OR and 95\% confidence intervals (Cis) are shown in Table 4. In summary, there was significantly increased odds of EP for those of older age (70-80 years, and $>80$ years) compared with the reference group of $<40$ years. In comparison with being in the least deprived category than those in categories 3, 2 or 1 (most deprived) also showed increased odds of EP, with an increasing trend as deprivation worsened. Smoking, either as a current or ex-smoker, showed increased odds of EP relative to never smokers, and an increased number of co-

\section{Table 1. GP practice characteristics}

\begin{tabular}{|l|c|c|}
\hline Characteristics & $\begin{array}{c}\text { Participating } \\
\text { practices }(\mathbf{N}=\mathbf{3 1}), \\
\mathbf{N}(\%)\end{array}$ & $\begin{array}{c}\text { Non-participating } \\
\text { practices }(\mathbf{N}=\mathbf{4 6}), \mathbf{N}(\%)\end{array}$ \\
\hline Practice list size & & $25(54)$ \\
\hline Small $(\leqslant 5000)$ & $10(32)$ & $17(37)$ \\
Medium (5001-10 000) & $11(36)$ & $4(9)$ \\
Large $(\geqslant 10001)$ & $10(32)$ &
\end{tabular}

\section{Practice SIMD ${ }^{\text {a }}$ deprivation quintiles}

\begin{tabular}{|l|c|c|}
\hline 1 Most deprived & $2(7)$ & $0(0)$ \\
2 & $2(7)$ & $8(17)$ \\
3 & $9(29)$ & $16(35)$ \\
4 & $9(29)$ & $11(24)$ \\
5 Least deprived & $9(29)$ & $11(24)$ \\
\hline Urban rural (six-fold) & $12(38)$ & $9(20)$ \\
\hline Large urban & $4(13)$ & $3(6)$ \\
Other urban & $1(3)$ & $4(9)$ \\
Accessible small towns & $5(16)$ & $4(9)$ \\
Rural small towns & $2(7)$ & $9(20)$ \\
Accessible rural & $7(23)$ & $17(37)$ \\
Remote rural & $5(16)$ & $14(30)$ \\
\hline Number of male/female GPs per practice \\
\hline Male preponderance
\end{tabular}

Table 2. Route to diagnosis by cancer type diagnosed between 2007 and 2013

\begin{tabular}{|c|c|c|c|c|c|}
\hline \multirow[b]{3}{*}{$\begin{array}{l}\text { Cancer } \\
\text { type }\end{array}$} & \multicolumn{5}{|c|}{ Route to diagnosis $(N=1802)$} \\
\hline & \multicolumn{4}{|c|}{$\begin{array}{c}\text { Non-emergency } \\
\text { presentation }(N=1437)\end{array}$} & \multirow{2}{*}{\begin{tabular}{|c|}
$\begin{array}{c}\text { Emergency } \\
\text { presentation } \\
(N=365)\end{array}$ \\
GP/A\&E)
\end{tabular}} \\
\hline & GP USC ${ }^{a}$ & $\begin{array}{c}\text { GP } \\
\text { urgent }^{b}\end{array}$ & $\begin{array}{c}\text { GP } \\
\text { routine }\end{array}$ & Other $^{d}$ & \\
\hline & $N(\%)$ & $N(\%)$ & $N(\%)$ & $N(\%)$ & $N(\%)$ \\
\hline Breast & $180(44)$ & $118(29)$ & $68(17)$ & $26(6)$ & $16(4)$ \\
\hline Colorectal & $108(27)$ & $92(23)$ & $65(16)$ & $15(4)$ & $122(30)$ \\
\hline Lung & $105(29)$ & $65(18)$ & $23(6)$ & $30(8)$ & $145(39)$ \\
\hline Melanoma & 32 (39) & 31 (37) & $11(13)$ & 7 (8) & $2(2)$ \\
\hline Prostate & $118(33)$ & $80(23)$ & $111(31)$ & $24(7)$ & $21(6)$ \\
\hline \multirow[t]{2}{*}{ Upper GI } & $46(25)$ & $56(30)$ & $17(9)$ & 9 (5) & $59(32)$ \\
\hline & $589(33)$ & $442(25)$ & $295(16)$ & $111(6)$ & $365(20)$ \\
\hline $\begin{array}{l}\text { Abbreviation: } \\
a_{\text {a }} \text {, USC }=\mathrm{GP} \\
\mathrm{b}_{\mathrm{GP}} \text { urgent }= \\
{ }^{c_{G P} \text { routine }=} \\
{ }^{d_{O} \text { ther }=\text { diag }}\end{array}$ & rgent referra & titioner. & 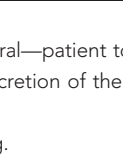 & 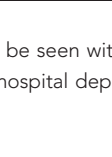 & $\begin{array}{l}\text { in } 2 \text { weeks. } \\
\text { rtment. }\end{array}$ \\
\hline
\end{tabular}


Table 3. Patient and general practitioner (GP) sociodemographic characteristics non-emergency and emergency presentations with associated emergency presentation (EP) subroutes

EP subroutes

\begin{tabular}{|c|c|c|c|c|c|c|}
\hline Characteristics & $\begin{array}{c}\text { Non-emergency } \\
\text { presentation, } \\
N(\%)\end{array}$ & $\begin{array}{c}\text { Emergency } \\
\text { presentation, } \\
N(\%)\end{array}$ & $\begin{array}{l}\text { No prior primary } \\
\text { care contact } \\
\text { before EP, } N(\%)\end{array}$ & $\begin{array}{c}\text { EP occurred within } \\
\text { an appropriate } \\
\text { episode of care, } \\
N(\%)\end{array}$ & $\begin{array}{l}\text { EP occurred while } \\
\text { waiting for a } \\
\text { relevant secondary } \\
\text { care appointment, } \\
N(\%)\end{array}$ & $\begin{array}{c}\text { EP occurred } \\
\text { following a missed } \\
\text { opportunity for } \\
\text { earlier diagnosis, } \\
N(\%)\end{array}$ \\
\hline Age & $N=1437$ & $N=365$ & $N=94$ & $N=115$ & $N=78$ & $N=50$ \\
\hline $\begin{array}{l}<40 \\
40-49 \\
50-59 \\
60-69 \\
70-80 \\
>80\end{array}$ & $\begin{array}{l}38(2.6) \\
113(7.9) \\
203(14.1) \\
384(26.7) \\
456(31.7) \\
243(16.9)\end{array}$ & $\begin{array}{r}3(<1) \\
12(3.3) \\
45(12.3) \\
88(24.1) \\
126(34.5) \\
91(24.9)\end{array}$ & $\begin{array}{c}0(0) \\
3(3.2) \\
12(12.8) \\
23(24.5) \\
31(33.0) \\
24(26.6)\end{array}$ & $\begin{array}{c}1(0.9) \\
3(2.6) \\
13(11.3) \\
32(27.8) \\
35(30.4) \\
31(27.0)\end{array}$ & $\begin{array}{c}2(2.6) \\
3(3.8) \\
9(11.5) \\
16(20.5) \\
28(35.9) \\
20(25.6)\end{array}$ & $\begin{aligned} 0(0) \\
2(4.0) \\
4(8.0) \\
12(24.0) \\
25(50.0) \\
7(14.0)\end{aligned}$ \\
\hline Gender & $N=1437$ & $N=365$ & $N=94$ & $N=115$ & $N=78$ & $\mathbf{N}=\mathbf{5 0}$ \\
\hline $\begin{array}{l}\text { Male } \\
\text { Female }\end{array}$ & $\begin{array}{l}728(50.7) \\
709(49.3)\end{array}$ & $\begin{array}{l}193(52.9) \\
172(47.1)\end{array}$ & $\begin{array}{l}61(64.9) \\
33(35.1)\end{array}$ & $\begin{array}{l}64(55.7) \\
51(44.3)\end{array}$ & $\begin{array}{l}37(47.4) \\
41(52.6)\end{array}$ & $\begin{array}{l}15(30.0) \\
35(70.0)\end{array}$ \\
\hline Deprivation & $N=1437$ & $N=365$ & $N=94$ & $N=115$ & $N=78$ & $N=50$ \\
\hline $\begin{array}{l}1 \text { Most deprived } \\
2 \\
3 \\
4 \\
5 \text { Least deprived }\end{array}$ & $\begin{array}{l}100(7.0) \\
178(12.4) \\
275(19.1) \\
399(27.8) \\
485(33.8)\end{array}$ & $\begin{array}{l}34(9.3) \\
60(16.4) \\
78(21.4) \\
99(27.1) \\
94(25.8)\end{array}$ & $\begin{array}{c}9(9.6) \\
21(22.3) \\
21(22.3) \\
22(23.4) \\
21(22.3)\end{array}$ & $\begin{array}{c}9(7.8) \\
14(12.2) \\
25(21.7) \\
29(25.2) \\
38(33.0)\end{array}$ & $\begin{aligned} 11 & (14.1) \\
14 & (17.9) \\
7 & (9.0) \\
25 & (32.1) \\
21 & (26.9)\end{aligned}$ & $\begin{array}{c}3(6.0) \\
5(10.0) \\
15(30.0) \\
17(34.0) \\
10(20.0)\end{array}$ \\
\hline Rurality sixfold & $N=1437$ & $N=365$ & $N=94$ & $N=115$ & $N=78$ & $N=50$ \\
\hline $\begin{array}{l}1 \text { Large urban } \\
2 \\
3 \\
4 \\
5 \\
6 \text { Remote rural }\end{array}$ & $\begin{array}{l}595(41.4) \\
204(14.2) \\
115(8.0) \\
200(13.9) \\
161(11.2) \\
162(11.3)\end{array}$ & $\begin{array}{c}154(42.2) \\
63(17.3) \\
32(8.7) \\
38(10.4) \\
39(10.7) \\
39(10.7)\end{array}$ & $\begin{aligned} 44 & (46.8) \\
12 & (12.8) \\
6 & (6.4) \\
13 & (13.8) \\
7 & (7.4) \\
12 & (12.8)\end{aligned}$ & $\begin{array}{l}41(35.7) \\
22(19.1) \\
15(13.0) \\
12(10.4) \\
11(9.6) \\
14(12.2)\end{array}$ & $\begin{aligned} 41 & (52.6) \\
12 & (15.4) \\
4 & (5.1) \\
4 & (5.1) \\
15 & (19.2) \\
2 & (2.6)\end{aligned}$ & $\begin{aligned} & 16(32.0) \\
& 13(26.0) \\
& 6(12.0) \\
& 7(14.0) \\
& 3(6.0) \\
& 5(10.0)\end{aligned}$ \\
\hline Smoking status & $N=1428$ & $N=363$ & $N=94$ & $N=113$ & $N=78$ & $N=50$ \\
\hline $\begin{array}{l}\text { Current smoker } \\
\text { Ex-smoker } \\
\text { Never smoked }\end{array}$ & $\begin{array}{l}351(24.6) \\
557(39.0) \\
520(36.4)\end{array}$ & $\begin{array}{r}128(35.3) \\
144(39.7) \\
91(25.1)\end{array}$ & $\begin{array}{l}31(33.0) \\
42(44.7) \\
21(22.3)\end{array}$ & $\begin{array}{l}38(33.6) \\
49(43.4) \\
26(23.0)\end{array}$ & $\begin{array}{l}30(38.5) \\
23(29.5) \\
25(32.1)\end{array}$ & $\begin{array}{r}18(36.0) \\
23(46.0) \\
9(18.0)\end{array}$ \\
\hline Ethnicity & $N=617$ & $N=149$ & $N=33$ & $N=49$ & $N=41$ & $N=19$ \\
\hline $\begin{array}{l}\text { White } \\
\text { Other ethnic } \\
\text { background }\end{array}$ & $\begin{array}{l}583(94.5) \\
34(5.5)\end{array}$ & $\begin{array}{r}133(89.3) \\
16(10.7)\end{array}$ & $\begin{array}{r}29(87.9) \\
4(12.1)\end{array}$ & $\begin{array}{r}43(87.8) \\
6(12.2)\end{array}$ & $\begin{array}{c}37(90.2) \\
4(9.8)\end{array}$ & $\begin{array}{r}17(89.5) \\
2(10.5)\end{array}$ \\
\hline GP list size & $N=1437$ & $N=365$ & $N=101$ & $N=115$ & $N=78$ & $\mathbf{N}=\mathbf{5 0}$ \\
\hline $\begin{array}{l}\text { Small } \\
\text { Medium } \\
\text { Large }\end{array}$ & $\begin{array}{l}172(12.0) \\
468(32.5) \\
797(55.5)\end{array}$ & $\begin{array}{r}39(10.7) \\
132(36.2) \\
194(53.1)\end{array}$ & $\begin{array}{l}16(15.8) \\
44(43.6) \\
41(40.6)\end{array}$ & $\begin{array}{l}14(12.2) \\
28(24.3) \\
73(63.5)\end{array}$ & $\begin{array}{c}3(3.8) \\
31(39.7) \\
44(56.4)\end{array}$ & $\begin{array}{c}3(6.0) \\
20(40,0) \\
27(54.0)\end{array}$ \\
\hline $\begin{array}{l}\text { GP male : female } \\
\text { ratio }\end{array}$ & $N=1437$ & $N=365$ & $N=101$ & $N=115$ & $N=78$ & $N=50$ \\
\hline $\begin{array}{l}\text { Equal } \\
\text { Male } \\
\text { preponderance } \\
\text { Female } \\
\text { preponderance }\end{array}$ & $\begin{array}{l}237(16.5) \\
285(19.8) \\
915(63.7)\end{array}$ & $\begin{array}{l}60(16.4) \\
57(15.6) \\
248(67.9)\end{array}$ & $\begin{array}{l}17(16.8) \\
14(13.9) \\
70(69.3)\end{array}$ & $\begin{array}{l}25(21.7) \\
24(20.9) \\
66(57.4)\end{array}$ & $\begin{array}{r}8(10.3) \\
10(12.8) \\
60(76.9)\end{array}$ & $\begin{array}{l}8(16.0) \\
8(16.0) \\
34(68.0)\end{array}$ \\
\hline GP deprivation & $N=1437$ & $N=365$ & $N=101$ & $N=115$ & $N=78$ & $N=50$ \\
\hline $\begin{array}{l}1 \text { Most deprived } \\
2 \\
3 \\
4 \\
5 \text { Least deprived }\end{array}$ & $\begin{array}{c}95(6.6) \\
68(4.7) \\
586(40.8) \\
347(24.0) \\
341(23.7)\end{array}$ & $\begin{array}{c}31(8.5) \\
22(6.0) \\
131(35.9) \\
84(23.0) \\
97(26.6)\end{array}$ & $\begin{array}{c}6(5.9) \\
8(7.9) \\
45(44.6) \\
19(18.8) \\
23(22.8)\end{array}$ & $\begin{array}{c}14(2.2) \\
4(3.5) \\
35(30.4) \\
32(27.8) \\
30(26.1)\end{array}$ & $\begin{array}{c}7(9.0) \\
5(6.4) \\
27(34.6) \\
15(19.2) \\
24(30.8)\end{array}$ & $\begin{array}{c}3(6.0) \\
5(10.0) \\
18(36.0) \\
13(26.0) \\
11(22.0)\end{array}$ \\
\hline $\begin{array}{l}\text { GP rurality } \\
\text { two-fold }\end{array}$ & $N=1437$ & $N=365$ & $N=101$ & $N=115$ & $N=78$ & $N=50$ \\
\hline $\begin{array}{l}1 \text { Urban } \\
2 \text { Rural }\end{array}$ & $\begin{array}{l}959(66.7) \\
478(33.3)\end{array}$ & $\begin{array}{l}254(70.0) \\
111(30.1)\end{array}$ & $\begin{array}{l}68(67.3) \\
33(32.7)\end{array}$ & $\begin{array}{l}74(64.3) \\
41(35.7)\end{array}$ & $\begin{array}{l}65(83.3) \\
13(16.7)\end{array}$ & $\begin{array}{l}32(64.0) \\
18(36.0)\end{array}$ \\
\hline
\end{tabular}

morbidities showed increased odds of EP relative to no comorbidity.

In the adjusted model, the strongest significant predictor of EP was having no prior primary care contact $(\mathrm{OR}=3.89$; CI 2.14 to 7.09). Other predictors that remained significant after adjustment were cancer type; specifically lung $(\mathrm{OR}=23.24$; CI 7.91-68.21), colorectal $(\mathrm{OR}=18.49$; $\mathrm{CI} 6.60-51.82)$ or upper GI cancer $(\mathrm{OR}=18.97$; CI 6.08-59.23) with the reference case being breast cancer. Also significant was non-white ethnicity $(\mathrm{OR}=2.78$; CI 1.27-6.06; Table 4). 
Table 4. Predictors of emergency presentation

\begin{tabular}{|c|c|c|c|c|c|}
\hline Factor & All presentations, $\mathbf{N}$ & $\begin{array}{c}\text { Non-emergency } \\
\text { presentation, } N(\%)\end{array}$ & $\begin{array}{c}\text { Emergency } \\
\text { presentation, } N(\%)\end{array}$ & $\begin{array}{l}\text { Unadjusted odds } \\
\text { ratio }(95 \% \mathrm{Cls})\end{array}$ & $\begin{array}{l}\text { Adjusted odds } \\
\text { ratio }^{a}(95 \% \mathrm{Cl})\end{array}$ \\
\hline Age & $N=1802$ & $N=1437$ & $N=365$ & & \\
\hline$<40$ & 41 & $38(92.7)$ & $3(7.3)$ & Reference & Reference \\
\hline $50-59$ & 248 & 203 (81.9) & $45(18.1)$ & $2.81(0.83-9.50)$ & $0.72(0.15-3.39)$ \\
\hline $60-69$ & 472 & $384(81.4)$ & $88(18.6)$ & $2.90(0.88-9.62)$ & $0.58(0.13-2.61)$ \\
\hline $70-80$ & 582 & $456(78.4)$ & $126(21.6)$ & $3.50(1.06-11.53)$ & $0.71(0.16-3.20)$ \\
\hline $\begin{array}{l}\text { Male } \\
\text { Female }\end{array}$ & $\begin{array}{l}921 \\
881\end{array}$ & $\begin{array}{l}728(79.0) \\
709(80.5)\end{array}$ & $\begin{array}{l}193(21.0) \\
172(19.5)\end{array}$ & $\begin{array}{c}\text { Reference } \\
0.92(0.73-1.15)\end{array}$ & \\
\hline Deprivation & $N=1802$ & $N=1437$ & $N=365$ & & \\
\hline 1 Most deprived & 134 & $100(74.6)$ & $34(25.4)$ & $1.75(1.21-2.74)$ & $1.29(0.55-3.01)$ \\
\hline 2 & 238 & $178(74.8)$ & $60(25.2)$ & $1.74(1.21-2.51)$ & $1.37(0.71-2.65)$ \\
\hline 3 & 353 & 275 (77.9) & 78 (22.1) & $1.46(1.05-2.05)$ & $1.46(0.75-2.81)$ \\
\hline Cancer type & $N=1802$ & $N=1437$ & $N=365$ & & \\
\hline Breast & 408 & $392(96.1)$ & $16(3.9)$ & Reference & Reference \\
\hline Colorectal & 402 & $280(69.7)$ & $122(30.3)$ & $10.68(6.20-18.38)$ & $18.49(6.60-51.82)$ \\
\hline Lung & 368 & $223(60.6)$ & 145 (39.4) & $15.93(9.27-27.39)$ & $23.24(7.92-68.21)$ \\
\hline Melanoma & 83 & 81 (97.6) & $2(2.4)$ & $0.61(0.14-2.68)$ & $1.04(0.11-9.63)$ \\
\hline Upper gastrointestinal & 187 & $128(68.4)$ & $59(31.6)$ & $11.29(6.28-20.32)$ & $18.97(6.08-59.23)$ \\
\hline Prostate & 354 & $333(94.1)$ & $21(5.9)$ & $1.55(0.79-3.01)$ & $1.55(0.44-5.50)$ \\
\hline Smoking status & $N=1791$ & $N=1428$ & $N=363$ & & \\
\hline Current smoker & 479 & $351(73.3)$ & $128(26.7)$ & $2.08(1.52-2.82)$ & $0.99(0.53-1.84)$ \\
\hline Ex-smoker & 701 & 557 (79.5) & $144(20.5)$ & $1.48(1.11-1.97)$ & $0.69(0.39-1.21)$ \\
\hline Never smoked & 611 & $520(85.1)$ & $91(14.9)$ & Reference & \\
\hline Yes & 1605 & $1345(83.8)$ & $260(16.2)$ & REFERENCE & \\
\hline No & 193 & $92(47.7)$ & $101(52.3)$ & $5.68(4.16-7.76)$ & $3.89(2.14-7.09)$ \\
\hline GP list size & $N=1802$ & $N=1437$ & $N=365$ & & \\
\hline Small & 211 & $172(81.5)$ & $39(18.5)$ & Reference & \\
\hline Medium & 600 & $468(78.0)$ & $132(22.0)$ & $1.244(0.84-1.85)$ & \\
\hline Large & 991 & $797(80.4)$ & $194(19.6)$ & $1.07(0.73-1.57)$ & \\
\hline GP male : female ratio & $N=1802$ & $N=1437$ & $N=365$ & & \\
\hline Equal & 297 & $237(79.8)$ & $60(20.2)$ & Reference & \\
\hline Male preponderance & 342 & $285(83.3)$ & 57 (16.7) & $0.79(0.53-1.18)$ & \\
\hline Female preponderance & 1163 & 915 (78.7) & $248(21.3)$ & $1.07(0.78-1.47)$ & \\
\hline GP deprivation & $N=1802$ & $N=1437$ & $N=365$ & & \\
\hline 1 Most deprived & 126 & 95 (75.4) & $31(24.6)$ & $1.15(0.72-1.82)$ & \\
\hline 2 & 90 & $68(75.6)$ & $22(24.4)$ & $1.14(0.67-1.93)$ & \\
\hline 3 & 717 & $586(81.7)$ & $131(18.3)$ & $0.79(0.59-1.10)$ & \\
\hline 4 & 431 & $347(80.5)$ & $84(19.5)$ & $0.85(0.61-1.18)$ & \\
\hline 5 Least deprived & 438 & 341 (77.9) & $97(22.1)$ & Reference & \\
\hline GP rurality twofold & $N=1802$ & $N=1437$ & $N=365$ & & \\
\hline 1 Urban & 1213 & $959(79.1)$ & $254(20.9)$ & Reference & \\
\hline 2 Rural & 589 & $478(81.2)$ & $111(18.8)$ & $0.88(0.68-1.12)$ & \\
\hline
\end{tabular}




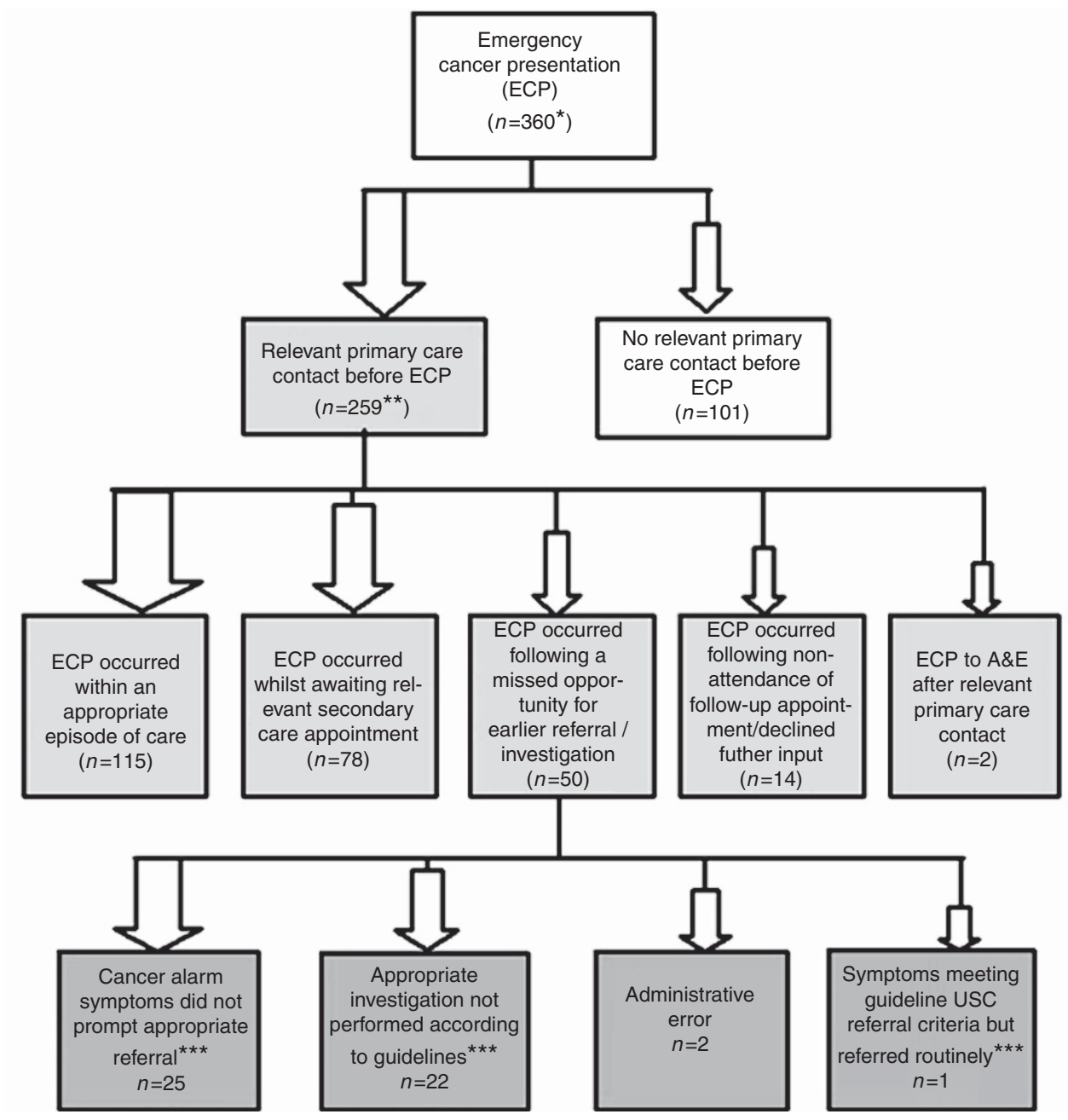

Figure 1. Interaction with primary care before ECP. *Unable to code five patients due to lack of content in consultations prior to their EP. **Relevant consultaions $=$ those that included any clinical signs and symptoms in the Scottish Referral Guidelines for Suspected Cancer that recommended investigation/referral. ${ }^{\star * \star}$ Scottish Government (2014).

Descriptive analysis of exploration of interaction with primary care before EP. Figure 1 illustrates how our detailed exploration of interaction with primary care before EP resulted in 360 patients being assigned a primary code most relevant to their EP (we were unable to apply a code to five EP patients due to lack of content in consultations prior to their EP). Proportionally this showed that most EP patients $(72 \%, n=259)$ had seen a GP in the lead-up to their EP. Of those seeing a GP in the lead-up to their EP $44 \%$ $(n=115)$ were admitted to hospital within an appropriate episode of care. A further $30 \%(n=78)$ of patients had been appropriately referred to secondary care but were admitted as emergencies in the interim between their referral and their appointment date or before an appointment date was received. Three per cent $(n=7)$ had defaulted a recommended follow-up GP appointment prior to EP. Similarly, seven patients (3\%) either refused or were reluctant for a secondary care investigation prior to their EP. Less than $1 \%$ had seen their GP before their EP to A\&E. There appeared however to be a genuine missed opportunity for possible earlier diagnosis in $19 \%(n=50)$ of emergency admissions. Table 5 describes in detail the context in which each EP in the sample occurred. For each of the eight EP contexts (detailed in Box 1), we describe the overall proportion of EPs occurring within each context, with a further subdivision to describe EP contexts by cancer site. The type of primary care interaction before the EP differs by cancer site, with breast and melanoma most likely to present direct to primary care (without prior interaction). There were nearly $20 \%$ of lung and upper GI where the EP followed a missed opportunity. Table 5 also includes the median (interquartile range) in days from first presentation to the EP that led to hospital admission and diagnosis.

Genuine missed opportunities for possible earlier diagnosis. Fifty patients (10 with upper-GI cancer; 13 with colorectal cancer; and 27 with lung cancer) who went on to present as an emergency had primary care diagnostic pathways that fell out-with standards set in Scottish urgent-suspected-cancer guidelines (SRGSC) (Table 6$)$. In $50 \%$ of cases $(n=25)$ this was because red flag symptoms (e.g., anaemia, altered bowel habit) were recorded in the primary care case notes but had not prompted a corresponding urgent referral. In a further 22 patients (44\%) symptoms recorded in GP records (e.g., persistent cough or dyspnoea) suggested an urgent chest X-ray could have been arranged earlier. For two patients (4\%) administrative errors had occurred: for one an investigation for referral appears not to have been sent from the practice, and for another an appointment for follow-up in secondary care did not appear to have been arranged.

EP patients were more likely to have three or more GP consultations compared with non-EP patients (Figure 2) with those in the 'missed opportunities' category presenting repeatedly to primary care with relevant symptoms, $72 \%(n=36 / 50)$ had six or more consultations. These are shown in Table 6 , which suggests 


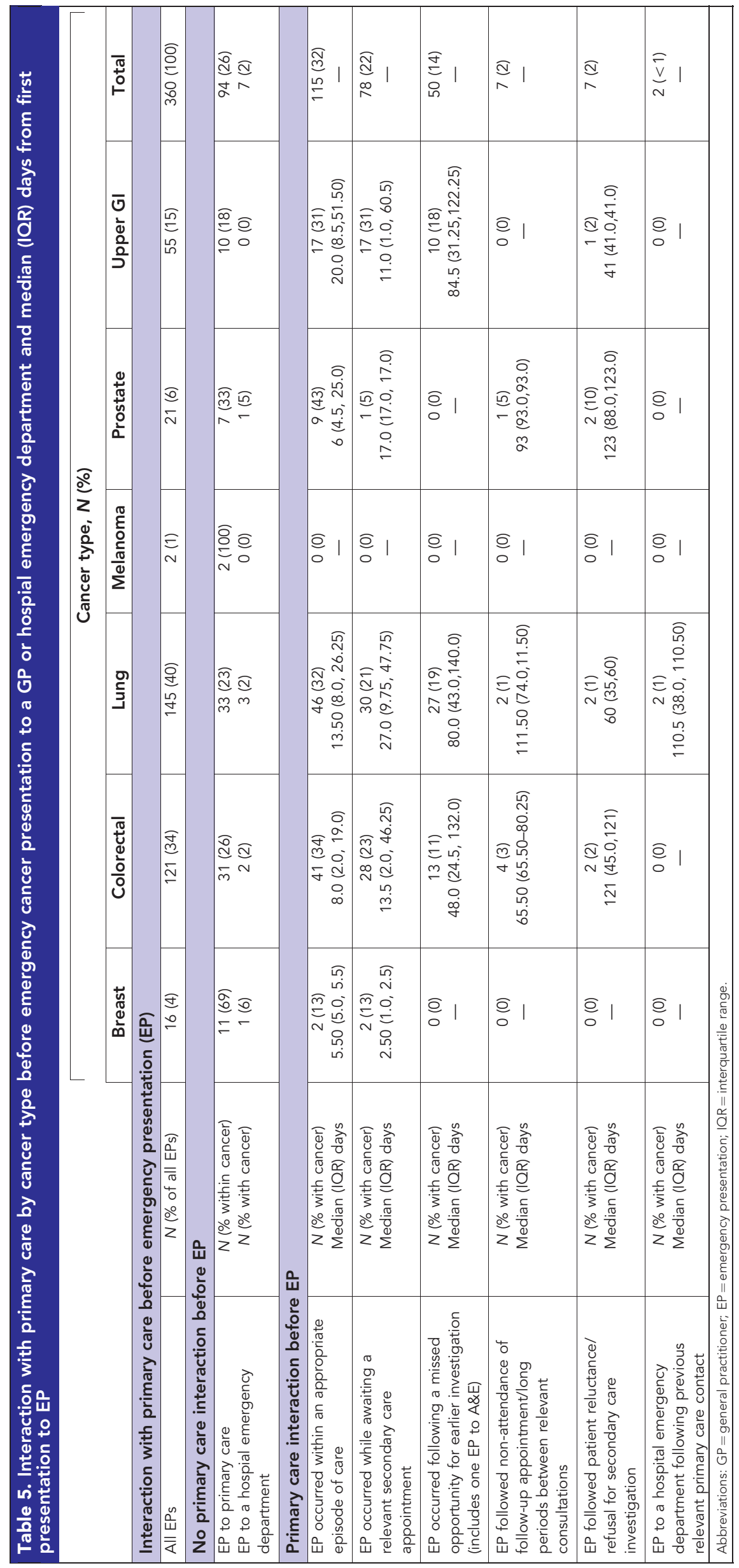




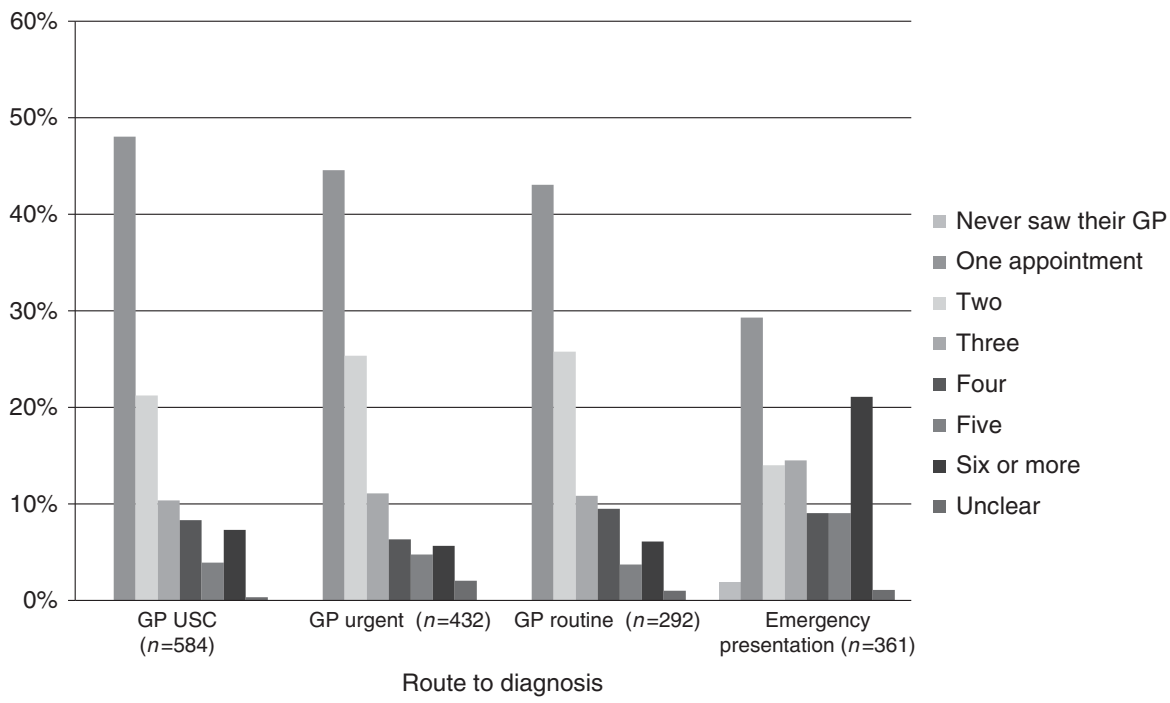

Figure 2. Number of GP appointments before the referral or admission that led to diagnosis.

Table 6. Missed opportunity for possible earlier diagnosis by cancer type and number of GP appointments before emergency presentation

\begin{tabular}{|l|c|c|c|c|}
\cline { 2 - 4 } & \multicolumn{4}{c|}{ Cancer site } \\
\hline $\begin{array}{l}\text { GP contact } \\
\text { before EP }\end{array}$ & Colorectal & Lung & Upper GI & $\begin{array}{c}\text { Total, } \\
\mathbf{n}(\%)\end{array}$ \\
\hline GP twice & 3 & 1 & 1 & $5(10)$ \\
\hline GP 4 times & 0 & 1 & 0 & $1(2)$ \\
\hline GP 5 times & 1 & 5 & 2 & $8(16)$ \\
\hline GP 6 or more times & 9 & 20 & 7 & $36(72)$ \\
\hline Total & 13 & 27 & 10 & $50(100)$ \\
\hline $\begin{array}{l}\text { Abbreviations: EP = emergency presentation; GP = general practitioner; GP = emergency } \\
\text { presentation. }\end{array}$
\end{tabular}

that earlier referral or investigation in these colorectal and upper GI cancer and lung cancers is achievable. Based on our context coding, we also looked at time in days from first presentation in primary care with relevant symptoms until the EP to primary care that led to admission and diagnosis. This showed that those in this category tended to have the longest median time period before diagnosis (Table 5).

\section{DISCUSSION}

Main findings. In total, 365 (20\%) of patients in our sample had their cancer diagnosed following an EP. The majority of these EPs could not have been averted by prior primary care contact; indeed, not having seen a GP $(n=103,28 \%)$ was by far the most powerful predictor of EP in our adjusted analysis. Qualitative context coding showed that $72 \%$ of patients $(n=259)$ did present to their GP in the lead up to their EP. Of these patients presenting in primary care beforehand, $44 \%(n=115)$ had appropriate care that met current guidelines for investigation and diagnosis of cancer. Nevertheless, we have shown that a small but significant proportion of emergency cancer diagnoses with prior GP contact present while awaiting secondary care intervention $(n=78,30 \%)$ or represent genuine, missed opportunities $(n=50,19 \%)$ for earlier referral within primary care.

Strengths and limitations. Previous studies have been able to assess trends and associations with respect to emergency cancer presentations and primary care interaction. To the best of our knowledge, this is the first study to use the approach of modelling predictors of EP of cancer while adjusting for actual prior primary care contact and also being able to interpret our model in the context of rich patient level data from individual cases. The study was based on a relatively large random sample comprising patients with six common cancers. One non-clinically trained researcher adhered strictly to referral criteria in Scottish Guidelines to apply context codes that minimised bias favouring GPs. Finally, the demographic characteristics of our sample, and the proportion of cancers diagnosed as an emergency, are similar to those reported within large national data sets providing reassurance that the sample is representative and the results meaningful for the wider UK.

Some limitations are acknowledged. The data are drawn from just one Scottish region; however, the population of Grampian is geographically and economically diverse reflecting the wider UK. Although, for the first time, we describe four broad categories of EP, our sample was not sufficiently large to explore differences between these groups, but data-pooling with other regions could enable this in future. For some cancers, especially melanoma and upper GI cancer, our sample comprises relatively fewer cases, but this would similarly challenge any study of this kind. It will be prudent to pool our data on these less common cancers with future samples to fully investigate their relationships to EPs.

Comparison with existing literature. Our proportion of EPs corresponded to previous reports (Bottle et al, 2012; Tsang et al, 2013; Abel et al, 2015; National Cancer Intelligence Network, 2015; Renzi et al, 2016). When we explored the impact of previous contact with primary care prior to EP in our multivariate model, it proved to be much the strongest predictor of EP, which mirrors the findings of a US study of 20000 people diagnosed with lung or colorectal cancer, where EP was significantly less likely for those seen in primary care (Sikka and Ornato, 2012). Being of an ethnicity other than white was similarly noted as a risk factor for EP in colorectal cancer by Wallace et al (2014) in their large study of over 90000 patients. Our finding that patients with colorectal, lung and upper GI cancer were significantly more likely to present as an emergency, compared with breast, melanoma or prostate cancer, was expected based on previous analyses of large, national data sets (Baughan et al, 2011; Elliss-Brookes et al, 2012; National Cancer Intelligence Network, 2015; Abel et al, 2015), and demonstrated that our sample for in-depth analysis of diagnostic context was representative. The aetiology and symptom expression of some cancers can mean that some may be easier (e.g., breast, 
melanoma, testicular, bladder) or harder (e.g., lung and stomach) to suspect (Lyratzopoulos et al, 2014). We explored this notion (Figure 1 and Table 2) and found support for it in the notably lower proportion of melanomas, breast and prostate cancers presenting as EPs.

Visiting a GP three or more times before referral is often considered to reflect an avoidable delay (Lyratzopoulos et al, 2014); however, previous studies have made this assumption without access to primary care case records. We have shown that those who appeared to have genuine, missed opportunities for earlier diagnosis in primary care (colorectal, lung and upper GI patients) frequently had six or more appointments before their EP. We also found that this group tended to have the longest median time period before the EP that led to diagnosis. Our findings support the case for future initiatives to promote earlier suspicion of cancer in primary care.

In Mitchell et al (2015b) reported a significant event analysis of practices in Northeast England and Southeast London only 3.6\% of EPs had no contact with a GP in the year preceding their emergency cancer presentation. Although the authors acknowledge that many emergency cancer presentations are complex, we believe that our methods enabled a more objective sample than in this study, which allowed GPs to select those cases which would be studied. Consequently, we suggest that our finding that $28 \%$ of patients had no relevant prior primary contact, may be a more representative figure. We do, however, agree that a greater understanding of complex presentations and potential future practice-level interventions have traction, but only as one part of a proportionate response to emergency cancer presentation.

In an observational study on ovarian cancer using primary care records Tate et al (2011) found that free text records, as distinct from coded records, contain important information on the severity of symptoms or on additional symptoms that have not been coded. Similarly, Ford et al (2013) when identifying patients with rheumatoid arthritis reported that diagnostic suspicions are frequently confined to text and that the use of electronic health records to create disease registers or assess quality of care will be misleading if free text information is not taken into consideration. This provides further justification for exploring the fine detail of primary care contact prior to EP.

Increased GP direct access to investigations has been implemented to some extent across the UK (NICE, 2015; Scottish Clinical Imaging Network, 2015). Also in both Scotland and England new referral guidelines with reduced thresholds have been published (Scottish Government, 2014; NICE, 2015). It seems likely that these initiatives may be contributing to apparent reductions in EP of cancer and we would support that these initiatives should continue to be promoted and implemented (National Cancer Intelligence Network, 2015).

Implications for policy and practice. EP is complex and does not have a single cause, and in some cases may be the most appropriate route to diagnosis. Our unique high definition analysis has revealed four broad categories of emergency cancer presentations. We recommend that research and health-care resource should be used proportionately across all four groups to identify the causes and, where necessary, deliver solutions.

First, there are patients who do not interact with GPs at all before they present as emergencies with features of their cancer. In this study $28 \%$ of EPs had no prior primary care contact. A study by the International Cancer Benchmarking Partnership (ICBP) found that people over 50 years of age in the UK compared with other highincome countries had lower awareness that the risk of cancer increases with age, and had more perceived barriers to symptomatic presentation (e.g., worrying about wasting the doctor's time, embarrassment and fear of what the GP might find; Forbes et al, 2013). Not recognising the seriousness of symptoms doubled the risk of delayed diagnosis and is likely to contribute to late stage at diagnosis and the poorer cancer survival rates in the UK compared with other similarly developed countries. Common symptoms associated with delay included urinary difficulties, change of bowel habit, systemic symptoms (fatigue, weight loss and loss of appetite) and skin symptoms (Forbes et al 2014). In light of this new information educational and awareness raising strategies about the benefits of early presentation with potential cancer features, such as the 'Be Clear on Cancer' campaign in England (Cancer Research UK (CRUK), 2016a) and 'Detect Cancer Early' in Scotland (Scottish Government, 2015), to raise public awareness of cancer symptoms are likely to have the most traction here, with benefits likely to be maximised by focusing on high-risk populations over 50 years, particular cancers and under-recognised symptoms.

Second, genuine missed opportunities for earlier diagnosis within primary care seemed to be rooted in non-adherence to referral guidelines by GPs, or where GPs had not recognised the significance of the content of previous consultations, for example, repeated consultations with the same symptom. In this study $19 \%$ of EPs with prior GP contact appeared to represent genuine missed opportunities for earlier diagnosis in primary care. The ICBP survey data have also provided some insight into why this may be. It found that UK primary care physicians showed the lowest readiness to refer or investigate a patient's symptoms at the first opportunity; this correlated with low cancer survival rates. UK primary care physicians also felt stronger about their role in protecting patients from too many tests, and in preventing specialists from being overloaded (CRUK, 2016b).

In their work on understanding missed opportunities for a more timely diagnosis, Lyratzopoulos et al (2015) call for more multidisciplinary work targeting factors in three phases of the diagnostic process where missed opportunities may occur and offer a conceptual foundation that builds on current approaches for the development of future interventions. CRUK's Facilitator programme that aims to support health-care professionals and relevant organisations to improve early cancer diagnosis is also likely to be of benefit here (CRUK, 2016c).

Third, there are those patients whose EP occurred in the interval between GP referral and being seen in secondary care, which occurred in $30 \%$ of the cases of EP with prior GP contact included in this study. For some this was within days of referral, but for others it occurred during a longer wait where patients' condition deteriorated while waiting to be seen in hospital. This may be partly due to secondary care system delays as, compared with other countries, UK GPs waited longer for results of tests such as CT and ultrasound scans (CRUK, 2016c). This suggests that there is scope for improvement in the system surrounding, and supporting, UK GPs in diagnosing cancer early. Existing initiatives, such as those described above, i.e., increasing direct GP access to investigations and lowering referral thresholds are again likely to be beneficial here especially for lung, colorectal and upper GI cancers, with some evidence that such approaches are already permitting gains in England (National Cancer Intelligence Network, 2015; NICE, 2015). In Scotland, the Scottish Government (2016) is committed to continuing the DCE awareness campaigns and providing support for earlier diagnosis through national screening programmes with a focus on hard-to-reach groups, and, driven by the findings of the ICBP, are providing greater investment to support swifter access to diagnostics for individuals with unidentified malignancies.

Fourth, and perhaps most complex of all, according to our context coding we classified about one-third of EPs as being within an appropriate episode of care. In this study $44 \%$ of EPs appeared to occur within appropriate episodes of care. This is a diverse group of patients. For some it is likely that aspects of patient and system delays contributed. However, in other cases the clinical care would have appeared to represent the best possible route to diagnosis for individual patients. 


\section{CONCLUSION}

Our analysis reveals that EP of cancer is complex and our novel approach has afforded new insights. GPs can be to blame, but not as often as supposed, and patient delay and sluggish referral pathways may also contribute. All should be tackled proportionately. However, sometimes EP affords individual patients the best chance of rapid treatment and cure and does not always represent failure. We suggest a proportionate and forward-looking program of emergency cancer presentation research and policy.

\section{ACKNOWLEDGEMENTS}

We would like to thank all participating general practices for taking part in this evaluation. The study was funded by the Chief Scientist's Office of the Scottish Government.

\section{CONFLICT OF INTEREST}

The authors declare no conflict of interest.

\section{AUTHOR CONTRIBUTIONS}

PM and AL designed the study. PM, AJL and SMS were members of the advisory steering group. MET and SF assisted with statistical analysis. All authors contributed to, read and approved the final manuscript.

\section{REFERENCES}

Abel G, Shelton J, Johnson S, Elliss-Brookes L, Lyratzopoulos G (2015) Cancer-specific variation in emergency presentation by sex, age and deprivation across 27 common and rarer cancers. Br J Cancer 112: S129-S136.

Barrett J, Hamilton W (2008) Pathways to the diagnosis of lung cancer in the UK: a cohort study. BMC Fam Pract 9: 31.

Baughan P, Keatings J, O'Neil B (2011) Urgent suspected cancer referrals from general practice: audit of compliance with guidelines and referral outcomes. Br J Gen Pract 61: e700-e706.

BBC (2016) Alarm symptoms missed in bowel cancer emergency patients, 28 September 2016. Available at: http://www.bbc.co.uk/news/health37493706.

Bottle A, Tsang C, Parsons C, Majeed A, Soljak M, Aylin P (2012) Association between patient and general practice characteristics and unplanned first-time admissions for cancer: observational study. Br J Cancer 107(8): 1213-1219.

Brewster DH, Clark DI, Stockton DL, Munro AJ, Steele RJC (2011) Characteristics of patients dying with 30 days of diagnosis of breast or colorectal cancer in Scotland, 2003-2007. Br J Cancer 104: 60-67.

Cancer Research UK (CRUK) (2016a) Be clear on cancer. Available at: http:// www.cancerresearchuk.org/health-professional/early-diagnosis-activities/ be-clear-on-cancer.

Cancer Research UK (CRUK) (2016b) International Cancer Benchmarking Partnership (ICBP) Evidence for Policy and Practice. Available at http:// www.cancerresearchuk.org/sites/default/files/ icbp_showcase_report_web.pdf.

Cancer Research UK (CRUK) (2016c) What is the Facilitator programme? Available at http://www.cancerresearchuk.org/health-professional/earlydiagnosis-activities/be-clear-on-cancer.

Collins N (2013) Tens of thousands of cancer patients visiting GP multiple times before referral. Available at http://www.telegraph.co.uk/news/health/ news/10276469/Tens-of-thousands-of-cancer-patients-visiting-GPmultiple-times-before-referral.html
Elliss-Brookes L, McPhail S, Ives A, Greenslade M, Shelton J, Hiom S, Richards M (2012) Routes to diagnosis for cancer-determining the patient journey using multiple routine data sets. Br J Cancer 107: 1220-1226.

Forbes LJL, Simon AE, Warburton F, Boniface D, Brain KE, Dessaix A (2013) Differences in cancer awareness and beliefs between Australia, Canada, Denmark, Norway, Sweden and the UK (the International Cancer Benchmarking Partnership): do they contribute to differences in cancer survival? Br J Cancer 108(2): 292-300.

Forbes L, Warburton F, Richard MA, Ramirez A (2014) Risk factors for delay in symptomatic presentation: a survey of cancer patients. Br J Cancer 111(3): 581-588.

Ford E, Nicholson A, Koeling R, Tate A, Carroll J, Axelrod L, Smith HE, Rait G, Davies KA, Petersen I, Williams T, Cassell JA (2013) Optimising the use of electronic health records to estimate the incidence of rheumatoid arthritis in primary care: what information is hidden in free text? BMC Med Res Methodol 13: 105.

Lyratzopoulos G, Neal RD, Barbiere JM, Rubin GP, Abel GA (2012) Variation in number of general practitioner consultations before hospital referral for cancer: findings from the 2010 National Cancer Patient Experience Survey in England. Lancet Oncol 13: 353-365.

Lyratzopoulos G, Wardle J, Rubin G (2014) Rethinking diagnostic delay in cancer: how difficult is the diagnosis? BMJ 349: g7400.

Lyratzopoulos G, Vedsted P, Singh H (2015) Understanding missed opportunities for more timely diagnosis of cancer in symptomatic patients after presentation. Br J Cancer 112(Suppl): S84-S91.

McArdle CS, Hole DJ (2004) Emergency presentation of colorectal cancer is associated with poor 5-year survival. Br J Surg 91: 605-609.

McPhail S, Elliss-Brookes L, Shelton J, Ives A, Greenslade M, Vernon S, Morris EJ, Richards M (2013) Emergency presentation of cancer and short-term mortality. Br J Cancer 109: 2027-2034.

Mitchell E, Pickwell-Smith B, Macleod U (2015a) Risk factors for emergency presentation with lung and colorectal cancers: a systematic review. BMJ Open 5: e006965.

Mitchell ED, Rubin G, Merriman L, Macleod U (2015b) The role of primary care in cancer diagnosis via emergency presentation: qualitative synthesis of significant event reports. Br J Cancer 112: S50-S56.

National Cancer Intelligence Network (2015) Routes to Diagnosis 2016-2013 Workbook. Public Health England. Available at: http://www.ncin.org.uk/ publications/routes_to_diagnosis.

NHS National Services Scotland (2016) Data dictionary, CHI number (Online). Available at: http://www.ndc.scot.nhs.uk/Dictionary-A-Z/ Definitions/index.asp?ID=128\&Title=CHI\%20Number3.

NICE (2015) Suspected cancer: recognition and referral. NICE Guideline 12. Available at nice.org.uk/guidance/ng12.

Renzi C, Lyratzopoulos G, Card T, Chu TPC, Macleod U, Rachet B (2016) Do colorectal cancer patients diagnosed as an emergency differ from non-emergency patients in their consultation patterns and symptoms? A longitudinal data-linkage study in England. Br J Cancer 115: 866-875.

Rubin G, Neal R, Abel G, Lyratzopoulos G (2013) Integrated research efforts are needed to better understand how to reduce the proportion of patients with cancer who are diagnosed as emergencies. Brit J Cancer 108(7): 1550-1551. Available at: http://doi.org/10.1038/bjc.2013.105.

Scottish Clinical Imaging Network (2015) A Scottish imaging pathway for primary care direct access to CT of chest/abdomen/pelvis for patients with unidentified suspected malignancy. Available at: http://www.scin.scot. nhs.uk/httpwww-scin-scot-nhs-ukworking-groupsscin-quality-improve ment/scin-national-direct-access-to-imaging/.

Scottish Executive Health Department (2007) Scottish Referral Guidelines for Suspected Cancer. SEHD: Edinburgh.

Scottish Executive Health Department (2009) Scottish Referral Guidelines for Suspected Cancer. SEHD: Edinburgh. Available at: http://www.gov.scot/ Resource/Doc/264972/0079346.pdf.

Scottish Government (2012) The Scottish Index of Multiple Deprivation. Available at: http://simd.scotland.gov.uk/publication-2012/.

Scottish Government (2014) Scottish referral guidelines for suspected cancer. SEHD, Edinburgh. Available at: http://www.shb.scot.nhs.uk/board/foi/ 2015/09/2015-319a.pdf.

Scottish Government (2015) Detect Cancer Early Programme. Available at: http://www.gov.scot/Topics/Health/Services/Cancer/Detect-Cancer-Early.

Scottish Government (2016) Beating Cancer: Ambition and Action. The Scottish Government: Edinburgh. Available at: http://www.gov.scot/ Publications/2016/03/9784/1. 
Sikka V, Ornato JP (2012) Cancer diagnosis and outcomes in Michigan EDs vs other settings. Am J Emerg Med 30(2): 283-292.

Tsang C, Bottle A, Majeed A, Aylin P (2013) Cancer diagnosed by emergency admission in England: an observational study using the general practice research database. BMC Health Serv Res 13: 308.

Tate AR, Martin AGR, Ali A, Cassell J (2011) Using free text information to explore how and when GPs code a diagnosis of ovarian cancer: an observational study using primary care records of patients with ovarian cancer. BMJ Open 1(1)): e000025.

Wallace D, Walker K, Kuryba A, Finan P, Scott N, van der Meulen J (2014) Identifying patients at risk of emergency admission for colorectal cancer. Br J Cancer 111: 577-580.
Wilcock A, Crosby V, Hussain A, McKeever TM, Manderson C, Farnan S, Freer S, Freemantle S, Littlewood F, Caswell G, Seymour J (2016) Lung cancer diagnosed following an emergency admission: mixed methods study of the management, outcomes and needs and experiences of patients and carers. Respir Med 114: 38-45.

This work is published under the standard license to publish agreement. After 12 months the work will become freely available and the license terms will switch to a Creative Commons AttributionNonCommercial-Share Alike 4.0 Unported License. 